\title{
Tuxpan: Análisis del sector agropecuario con la ampliación del puerto
}

Tuxpan: Analysis of the agricultural sector with the expansion of the port

\author{
Mateos Rocha Roberto Alejandro $^{1 \bowtie}$, Hernández Márquez Sabdy Greysi ${ }^{1}$
}

${ }^{1}$ Facultad de Ciencias Biológicas y Agropecuarias, Universidad Veracruzana. Campus Tuxpan

${ }^{凶}$ Autor para correspondencia: romateos@uv.mx

Recibido: $19 / 01 / 2016$

Aceptado: 15/06/2016

\begin{abstract}
RESUMEN
La modernización de los puertos en el Estado de Veracruz, incluyendo al Puerto de Tuxpan, incrementara los niveles de rendimiento y competencia en las empresas, principalmente para las Micro, Pequeñas y Medianas Empresas las cuales aportan el 52\% del Producto Interno Bruto y el $72 \%$ del empleo que se genera en el país y para el Estado de Veracruz el sector agropecuario representa el primer lugar en cuanto a unidades de producción esto significa que el Estado es principalmente Agropecuario por lo que es importante analizar el beneficio que representa la ampliación del Puerto de Tuxpán para dicho sector de la zona norte del estado de Veracruz con la finalidad de describir la evolución en el movimiento de carga que ha tenido el puerto, establecer el beneficio de las empresas del sector agropecuario con la ampliación del puerto y determinar si la ampliación de dicho puerto influirá en el desarrollo de zona así como en el sector agropecuario. Con dicho análisis se prevé un panorama alentador y puede verse beneficiado en el aumento de su producción ya que, al reducir los costos logísticos, obtendrán más ganancias. También al aumentar la producción de las agroempresas estas requerirán más mano de obra lo que beneficiara, al generar empleos en el sector rural.
\end{abstract}

Palabras claves: Sector rural, Logística, Beneficios, Tuxpan, Puerto.

\begin{abstract}
The modernization of the ports in the State of Veracruz, including the Port of Tuxpan, will increase the levels of performance and competition in companies, mainly for Micro, Small and Medium Enterprises which contribute $52 \%$ of the Gross Domestic Product and $72 \%$ of the employment that is generated in the country and for the State of Veracruz, the agricultural sector represents the first place in terms of production units. This means that the State is mainly Agricultural, so it is important to analyze the benefit of the expansion of the Port. of Tuxpán for said sector of the northern zone of the state of Veracruz with the purpose of describing the evolution in the cargo movement that the port has had, establishing the benefit of the companies of the agricultural sector with the expansion of the port and determining if the extension of said port will influence the development of the area as well as the agricultural sector. With this analysis, an encouraging scenario is foreseen and can be benefited in the increase of its production, since by reducing
\end{abstract}


logistics costs, they will obtain more profits. Also, by increasing the production of agribusinesses, these will require more labor, which will benefit, by generating jobs in the rural sector.

Keywords: Rural sector, Logistics, Benefits, desvantajas, Port.

\section{INTRODUCCIÓN}

De acuerdo con información de la Organización Marítima Internacional (OMI) el transporte vía marítima es el "Vehículo del Comercio Mundial" esto se debe a que el transporte marítimo es el más económico, seguro y con mayor capacidad de carga en el comercio mundial (Opazo, 2015).

Los mismos costos de transporte marítimo pueden influir en los precios de algunos mercados como el caso de productos agrícolas, que son muy susceptibles a los tiempos programados de transporte y a las tarifas que puede influir de manera significativa en los precios, como es el caso de cereales y oleaginosa que son transportados en grandes volúmenes (bulk), lo mismo se puede decir del factor logístico pues un manejo eficiente de los flujos de carga, influye también en los precios de ciertos mercados, es por estos factores que las empresas utilizan el transporte marítimo para mover su carga hacia los diferentes países con los que tienen relaciones comerciales (García, Méxicoxport, 2014).

Aproximadamente el $90 \%$ del comercio mundial se transporta por vía marítima, la operación de buques mercantes genera un ingreso aproximado de 380 billones de dólares, equivalente a cerca del $5 \%$ del valor total del comercio mundial (García, 2014). El sistema portuario mexicano cuenta con 97 puertos que operan actualmente a través de un sistema de concesiones por parte del gobierno federal, de los cuales 16 son administrados por la Secretaria de Comunicaciones y Transportes, 2 operados por
Fonatur, también 5 son administrados por gobierno Estatal y 1 es privado (SCT, 2015).

Nuestro estado cuenta con varias aduanas marítimas: Administración Portuaria Integral de Veracruz, una de las más importantes en México en el movimiento de carga contenerizada y de vehículos (Huchim, Ortíz, y Giani, 2015), y la Administración Portuaria Integral de Tuxpan que se inauguró en 1994 para importaciones de combustibles, productos petroleros y productos agrícolas a granel con infraestructura muy limitada y deficiente para el manejo del volumen de mercancía, ya que los muelles son de poca profundidad, lo que limita el acceso de buques más grandes y por tanto ha afectado directamente al poco movimiento de mercancía en el puerto. Cruz (2013) menciona que otro factor que afecta el poco movimiento de mercancía del puerto se debe a que no cuenta con la maquinaria adecuada para el embarque y desembarque de la mercancía en contenedores como lo son, las grúas de pórtico y la maquinaria adecuada para este tipo de carga. Por último no existen los patios especializados para cada tipo de mercancía.

De acuerdo con información de la Secretaría de Desarrollo Económico y Portuario (SEDECOP) la modernización de los puertos en el Estado de Veracruz, incluyendo al Puerto de Tuxpan, incrementara los niveles de rendimiento y competencia en las empresas ( Política Empresarial, 2014), principalmente para las Micro, Pequeñas y Medianas Empresas (MiPymes) las cuales aportan el $52 \%$ del Producto Interno Bruto (PIB) y el $72 \%$ del empleo que se genera en el país (ProMéxico, 2014) y para el Estado de 
Veracruz el sector agropecuario representa el primer lugar en cuanto a unidades de producción (SAGARPA, 2008) esto significa que el Estado es principalmente Agropecuario.

En referencia al Programa Nacional de Infraestructura 2014-2018 se estableció para el puerto Tuxpan una inversión para la construcción de una instalación portuaria de uso público el cual contempla la construcción de muelle público para contenedores y carga en general. La primera etapa se inició en el año 2013 con el inicio de este y la etapa finalizara en febrero del presente año, la etapa final del proyecto será en el año 2018 (PNI, 2014). Por lo anterior surge la necesidad de realizar un análisis del beneficio que representa la ampliación del Puerto de Tuxpán para el sector agropecuario de la zona norte del estado de Veracruz.

\section{MATERIALES Y MÉTODOS}

Para poder llegar al objetivo planeado fue necesario realizar una investigación cualitativa y cuantitativa, que consistió en recabar información de libros, periódicos, medios electrónicos y entrevistas al personal de la Administración Portuaria Integral de Tuxpan con la finalidad de describir la evolución en el movimiento de carga que ha tenido el puerto de Tuxpán, Veracruz, establecer el beneficio de las empresas del sector agropecuario con la ampliación del puerto y determinar si la ampliación del Puerto de Tuxpan influirá en el desarrollo de zona así como en el sector agropecuario, para conocer el beneficio que representa la ampliación del Puerto de Tuxpán para el sector agropecuario de la zona norte del estado de Veracruz.
El transporte marítimo beneficia empresas al tener mejores rendimientos, así como la apertura de nuevos puertos y ampliando la oferta de estos servicios marítimos (Gonzales Laxe, 2015). Los puertos de tercera generación no solo son conectores del comercio terrestre con el marítimo, ahora representan un centro logístico con nodos dinámicos en la compleja red comercio internacional. Las alianzas comerciales al igual que los Tratados de Libre Comercio entre dos o más países fomentan y fortalecen el comercio mundial (ProMéxico, 2010), también es una manera de eliminar las barreras arancelarias $y$ tener tasas preferenciales al momento de pagar los impuestos en cada país, de esta manera se operan rutas comerciales más amplias.

El puerto de Tuxpan se localiza al norte del estado de Veracruz, ubicado en la parte central del Golfo de México, colinda al Este con el Municipio de Poza Rica, al Oeste con Tihuatlán, al Norte con Cerro Azul, al Sur con Papantla y al Noroeste con Álamo Temapache. El Puerto de Tuxpan cuenta actualmente con un muelle fiscal de usos múltiples con dos tramos de atraque de 427 metros de longitud total, un muelle de 65 metros de longitud en la margen izquierda denominado "muelle de dragas", una bodega de tránsito fiscalizado de $3,600 \mathrm{~m} 2$, un patio de almacenamiento de $90,000 \mathrm{~m} 2$ y un muelle de pesca; un camino de acceso a la zona portuaria y 2 escolleras que constituyen las obras de protección (la escollera norte con 1,400 metros de longitud y la escollera sur con 940 metros de extensión) ambas estructuras tienen parte emergida y sumergida, asimismo, se cuenta con un eficiente señalamiento marítimo que permite una navegación segura a las embarcaciones que arriban al Puerto, incluyendo la navegación nocturna.

\section{RESULTADOS Y DISCUSIÓN}


Algunas de las características del puerto de Tuxpan con respecto a la ampliación del mismo son:

Certificación de calidad por la norma International Organización for Standardization (ISO) 9001:2000 formada por los organismos de normalización de la mayoría de los países y fijan los estándares mínimos de calidad a nivel internacional.

El título de concesión vigente otorgado a favor de la administración portuaria integral de Tuxpan S.A de C.V cuenta con un área de 6,407 has integradas por las áreas terrestres y marítimas, de las cuales comprenden a área tierra 58.6 has y 6,348.4 has de agua.

Cuadro 1. Principales características del canal de navegación, también las cargas operadas de acuerdo al ingreso de buques en las terminales establecidas.

\begin{tabular}{|c|c|c|c|c|c|c|}
\hline Terminal & UbicaciónCalado y & Buque & TRB & TPM & Calado & Eslora \\
\hline & Profundidad & Característico & & & (pies) & (m) \\
\hline & escolleras (pies) & & & & & \\
\hline & $(\mathbf{K m})$ & & & & & \\
\hline Terminales Marítimas & 4.8 & 33Carga General & 22,629 & 37,096 & 18 & 187 \\
\hline Transunisa & & & & & & \\
\hline API de Tuxpan & 4.5 & 33Granel Mineral & 16,344 & $4 \quad 27,572$ & 32 & 180 \\
\hline Transferencias & 4.2 & 33Granel & 22,155 & 537,679 & 32 & 188 \\
\hline Graneleras & & Agrícola & & & & \\
\hline Granelera & 3.7 & 33Granel & 22,165 & 537,384 & 33 & 204 \\
\hline Internacional de & & Agrícola & & & & \\
\hline Tuxpan & & & & & & \\
\hline Compañía Terminal de & 3.5 & 33Granel Mineral & 19,872 & 234,224 & 19 & 198 \\
\hline Tuxpan & & & & & & \\
\hline Feno Resinas & 4.2 & 33Fluidos & 8,124 & 412,441 & 24 & 139 \\
\hline
\end{tabular}

Una de las principales desventajas es la poca profundidad del canal de navegación que se encuentra en aproximadamente con 33 pies, de esta manera niega el acceso de buques de mayor capacidad. En el canal de navegación es donde se observa que bajo las condiciones actuales que opera el puerto de Tuxpan, el puerto se limita a recibir embarcaciones con eslora de hasta de 190 metros, esto también se debe a las dimensiones de la dársena de ciaboga. Por tanto para que las embarcaciones con eslora de 220 metros o más se necesita aumentar el ancho de la dársena de ciaboga de los 220 metros a 280 metros.

Cuenta con 6 posiciones de atraque, de las cuales dos de estas se utilizan para carga general, están a cargo de Administración Portuaria Integral de Tuxpan, las demás pertenecen al sector privado y se utilizan para gráneles agrícolas, minerales general suelta y fertilizantes. También el puerto cuenta con dos monoboyas que pertenecen a PEMEX y CFE. Y se ubican en el muelle de dragas posicionada en el margen izquierdo del rio que está enfrente de las oficinas administrativas y la segunda con capacidad para desembarcar dos buques se encuentra entre las instalaciones de Transferencia Granelera S.A de C.V y Granelera Internacional de Tuxpam, S.A de C.V. Las cuales están destinadas al amarre temporal de diferentes equipos navales como lo son grúas para la reparación de plataformas de perforación y extracción petrolera. 
Tienen una capacidad para recibir la cantidad de 221 embarcaciones por año con 7.6 millones de toneladas de carga de diferentes tipos.

La capacidad de almacenamiento abarca $156,964 \mathrm{~m}^{2}$ de patios que tienen una capacidad de 105,641 toneladas también esta otro patio de 45,000 TEU`s que es un patio operado por la empresa Tecomar. También se cuenta con bodegas 38,662 $\mathrm{m} 2$ de bodegas y $3,460 \mathrm{~m}^{2}$ de tanques. Un recinto portuario de $6,000 \mathrm{~m}^{2}$ que actualmente tiene tres tanques con capacidad de $1,200 \mathrm{~m}^{3}$ cada uno. Dentro de los proyectos que la empresa contempla es aumentar la capacidad actual e instalar 12 tanques en total con la misma capacidad de los instalados (Puerto de Tuxpan, 2012).

La más importante ventaja en la cercanía que tiene el puerto hacia la zona centro de México. Tuxpan es el puerto comercial más cercano a la capital del país y de su área metropolitana a partir de la nueva autopista México - Tuxpán hay 333 kilómetros, lo cual le da ciertas ventajas frente a Veracruz y Tampico-Altamira que son los principales puertos del golfo de México. La importancia radica en que el valle de México es el principal centro de consumo del país.

La zona Metropolitana del Valle de México desde su conformación es el principal y más grande asentamiento de México concentra actualmente más de veinte millones de habitantes concentra actualmente más de veinte millones de habitantes que a lo largo de su historia se ha visto sujeto a una ocupación que ha experimentado movimientos migratorios de aumento o disminución de la población en diferentes etapas, además del crecimiento natural, que se ha reflejado en un proceso de expansión urbana modificando el entorno y dando lugar a perdida de suelo rural vs suelo urbano. (Irma Escamilla Herrera, 2012)
La región centro de México la integran siete entidades federativas: el distrito federal, estado de México, Hidalgo, Morelos, Puebla, Tlaxcala y Querétaro, el cual concentra entre el 30 y 40 por ciento de empleo manufacturero y terciario del país, así como el $33 \%$ de la población nacional.

Es este espacio regional se ha manifestado una reorganización más flexible acorde con una red urbana ampliamente comunicada que permite el constante intercambio, modificando el comportamiento de empresas que ya no requieren determinados factores de localización, pues se van reduciendo costos de transporte y los procesos productivos cada vez se segmentan, para obtener ventajas económicas en distintas localizaciones y se generan economías de escala en su territorio regional. (Irma Escamilla Herrera, 2012)

$\mathrm{Su}$ actividad productiva ha generado una transformación de las áreas agrícolas que ha ido modificando el uso de suelo rural que por el constante crecimiento de la población se ha transformado en suelo de uso urbano con lo anterior y por este proceso de urbanización se intensifica la circulación de mercancías, ya que surge la necesidad de abastecer con recursos materiales (primarios) los procesos industriales para su transformación en productos finales, o simplemente por las necesidades de consumo tanto en perecederos así como productos industrializados. También los avances tecnológicos en los medios de transporte y comunicaciones tienen grandes ventajas al hacer que estos disminuyan los costos de transporte y de logística esto ha marcado tendencias de localización hacia las oportunidades que tienen la empresas para manejar costos más bajos, esto también se refleja en el tiempo porque esto se hacen más rápidos los traslados.

Asimismo, el Puerto de Tuxpan, tiene acceso a la costa este de EUA y Canadá, Centro y 
Sudamérica, Europa y África y sirve a las regiones socioeconómicas del Golfo y Centro Sur. Se vincula con los principales mercados del país por su excelente ubicación geográfica, infraestructura suficiente, servicios integrados, vías de comunicación que lo enlazan con las principales ciudades del país, tarifas portuarias competitivas, seguridad, mano de obra calificada que convierten a Tuxpan en una destacada opción para el manejo de Carga General, Granel Agrícola, Perecederos, Químicos Líquidos a Granel, Minerales a Granel, Derivados del Petróleo y construcción y mantenimiento de plataformas (Programa Maestro de Desarrollo Portuario, 2009-2011).

\section{CONCLUSIÓN}

Los beneficios más importantes que se pueden mencionar por la ampliación del puerto de Tuxpan en su primera etapa son:

Aprovechamiento que las agroempresas veracruzanas de la zona y de los estados vecinos que ya realicen importaciones o exportaciones y puedan hacerlo por el puerto de Tuxpan, Ver.

Para el sector agropecuario el panorama es alentador y se puede ver beneficiado en el aumento de su producción ya que al reducir los costos logísticos, obtendrán más ganancias. También al aumentar la producción de las agroempresas estas requerirán más mano de obra lo que beneficiara, al generar empleos en el sector rural.

Otro beneficio importante que tiene este proyecto de ampliación, es que generara más de dos mil empleos de manera directa e indirecta. También con la cercanía de las empresas que exportan sus productos, por los diferentes puertos del Golfo ahora tendrán otra opción para enviar sus productos a otros países de manera eficiente y segura.
Este proyecto de ampliación para el puerto, se enfoca principalmente en el movimiento de automóviles, así como la terminal especializada para contenedores, dentro de los contenedores se mueven diferentes tipos de mercancía, no necesariamente del sector agropecuario, sin embargo se espera que con la modernización y nueva infraestructura el puerto sea más atractivo para todas las empresas, y aumente el volumen de movimiento de carga para los diferentes sectores.

\section{LITERARTURA CITADA}

Política Empresarial. (2014). Sector Empresarial Motor De Desarrollo. Faena Política Empresarial.

Cruz, O. (2013). Conectividad, clave en el Plan Nacional de Infraestructura. México D.F: Grupo T-21.

García, R. (2014). Méxicoxport. Recuperado el 09 de Noviembre de 2015, de reto para actualizar el sector naval en México:

http://www.mexicoxport.com/noticias/10794 /90del-comercio-mundial-es-via-

maritima

Gonzales Laxe, F. (2015). Puertos y Transporte Marítimo: Ejes de una nueva articulación global.

Huchim, S., Ortíz, O., \& Giani, N. (2015).

Puertos Latinoamericanos. Revista Énfasis.

Irma Escamilla Herrera, C. S. (2012). LA ZONA METROPOLITANA DEL VALLE DE MÉXICO. Recuperado el 26 de AGOSTO de 2015, de http://www.ub.edu/geocrit/coloquio2012 /actas/07-I-Escamilla.pdf

Opazo, M. (10 de Noviembre de 2015). El Transporte Marítimo Mundial. Santiago, Chile.

Programa Maestro De Desarrollo Portuario Del Puerto De Tuxpan 2011-2016. 
(2013). Secretaría de Comunicaciones y Transportes.

Programa Nacional De Infraestructura. (2014). Programa Nacional De Infraestructura 2014-2018. México: Diario Oficial De La Federación.

ProMéxico. (2010). México y sus Tratados de Libre Comercio con otros Países. Recuperado el 17 de Noviembre de 2015 de http://mim.promexico.gob.mx/comercio/ mexico-y-sus-tratados-de-librecomercio-con-otros-paises.html

ProMéxico. (2014). PROMÉXICO. Recuperado el 10 de Noviembre de 2015, de PyMES, eslabón fundamental para el crecimiento en México: http://www.promexico.gob.mx/negociosinternacionales/pymes-eslabon- fundamental-para-el-crecimiento-enmexico.html

Puerto de Tuxpan. (2012). Puerto de Tuxpan. Recuperado el 30 de Septiembre de 2015, de http://puertotuxpan.com.mx/ultimasnoticias/96-el-puerto-de-tuxpan/125smart-pass-sa-de-cv-inaugurainstalacion-en-el-puerto-de-tuxpan SAGARPA. (2008). DIAGNÓSTICO DEL . Veracruz: Servicios y Asesoría La Cumbre, S.A. de C.V.

SCT:Secretaria De Comunicaciones Y Transportes. (2015). Secretaría de Comunicaciones y Transportes. Recuperado el 5 de Noviembre de 2015, de http://www.sct.gob.mx/puertos-ymarina/puertos-de-mexico/

Usted es libre para Compartir — copiar y redistribuir el material en cualquier medio o form ato- y Adaptar el documento —remezclar, transformar y crear a partir del material- para cualquier propósito, incluso para fínes comerciales, siempre que cumpla la condición de:

Atribución: Usted debe dar crédito a la obra original de manera adecuada, proporcionar un enlace a la licencia, e in dicar si se han realizado cam bios. Puede hacerlo en cualquier form a razonable, pero no de form a tal que sugiera que tiene el apoyo del licenciante o lo recibe por el uso que hace de la obra. 